International Mathematical Forum, Vol. 8, 2013, no. 19, 921 - 927

HIKARI Ltd, www.m-hikari.com

\title{
The Generalization of GCD Matrices
}

\author{
HAN Haiqing ${ }^{1}$ and ZHU Siru ${ }^{2}$
}

1. School of Mathematics and Physics

Hubei Polytechnic University

Huangshi 435003, Hubei, China

2. Department of the Basics, AFRA

Wuhan 430019, Hubei, China

Copyright (C) 2013 HAN Haiqing and ZHU Siru. This is an open access article distributed under the Creative Commons Attribution License, which permits unrestricted use, distribution, and reproduction in any medium, provided the original work is properly cited.

\begin{abstract}
Let $S_{1}, S_{2}, \cdots, S_{n}$ be $n$ finite sets of positive integers and $S=S_{1} \times S_{2} \times \cdots \times S_{n}$. We have got a $q \times q$ matrix $\langle S\rangle=$ $=\left(\operatorname{gcd}\left(d_{i}, d_{j}\right)\right)_{q \times q}$, where $s_{i j}=\operatorname{gcd}\left(d_{i}, d_{j}\right)$. In this paper, we study the bounds of the determinant of $\langle S\rangle$, and the value of the determinant of them in special condition. Finally, we generalize the GCD matrices to the direct product of general posets and obtain some results.
\end{abstract}

Keywords: the GCD matrix; the meet semi-lattice; the generalized Euler's function; the mobius inversion.

\section{Introduction}

The greatest common divisor matrices are a kind of special matrices defined in the positive integer set. And the properties of their determinants have been always the hot research. Beslin and Ligh have defined the greatest common divisor matrix ${ }^{[1]}$ on the positive integer set $S=\left\{x_{1}, x_{2}, \cdots, x_{n}\right\}$, take as $\langle S\rangle=\left(s_{i j}\right)_{n \times n}$,

$s_{i j}=\operatorname{gcd}\left(x_{i}, x_{j}\right)$, it is shorten to the GCD matrix and to proved $\langle S\rangle$ that is positive 
definite. In [2] has proved: if $S$ is the FC set then $\operatorname{det}\langle S\rangle=\phi\left(x_{1}\right) \phi\left(x_{2}\right) \cdots \phi\left(x_{n}\right)$, where $\phi$ is the Euler function. In [3], it has defined as the matrix $\langle S\rangle_{f}=\left(s_{i j}\right)_{n \times n}$, $s_{i j}=f\left(\operatorname{gcd}\left(x_{i}, x_{j}\right)\right)(1 \leq i, j \leq n)$. H.J.S.Smith has proved that if $S$ is FC set, then ${ }^{[4]}$ $\operatorname{det}\langle S\rangle_{f}=(f * \mu)\left(x_{1}\right)(f * \mu)\left(x_{2}\right) \cdots(f * \mu)\left(x_{n}\right)$. Where $f * \mu$ is the convolution. In [5-6], some good results have been obtained. In this paper, we have gotten some new generalization in this paper for the GCD matrix. In order to the convenient for the introduction, it is defined as follows.

In[5], It is the definition about the meet semi-lattice, the meet matrix LC and MC set, and LC and MC are the corresponding generalization of FC and GCDC set.

Definition 1 let $S$ be a subset of meet semi-lattice $(U, p)$, defined as $\Psi_{S, f}\left(x_{j}\right)=f\left(x_{j}\right)-\sum_{x_{i} p x_{j}, x_{i} \neq x_{j}} \Psi_{S, f}\left(x_{i}\right)$, its value is 0 when the sum item is empty, where $f$ is the real function in $U, \Psi_{S, f}$ be called the generalized Euler function on $S$.

Remark 1 By the Mobius inversion ${ }^{[7]}$, $f\left(x_{j}\right)=\sum_{x_{i} p x_{j}} \Psi_{S, f}\left(x_{i}\right)$.

Now we have generalized the concept of GCD matrix. $\forall 1 \leq i \leq n$, let $p_{i}$ be the partially ordered relation on $S_{i}$, that is

$\forall x_{i}, y_{i} \in S_{i}, x_{i} p_{i} y_{i} \Leftrightarrow x_{i} \mid y_{i}$. Then $\left(S_{i}, p_{i}\right)$ is the poset. So $\left(S_{i}, p_{i}\right)$ is a subset of the meet semi-lattice. And let $S=S_{1} \times S_{2} \times \cdots \times S_{n}$, defined a partially ordered relation $p$ for $S . \forall x, y \in S, x p y \Leftrightarrow x_{i} \mid y_{i}, 1 \leq i \leq n$. $(S, p)$ is a finite poset and the meet semi-lattice too. $\forall x, y \in S$, wo have the two conclusions:

1) $x \mid y \Leftrightarrow x p y, x$ is called the divisor of $y$;2) $\operatorname{gcd}(x, y)=\prod_{i=1}^{n} \operatorname{gcd}\left(x_{i}, y_{i}\right)$. Then

the meet matrix on $S$ is $\langle S\rangle=\left(\operatorname{gcd}\left(d_{i}, d_{j}\right)\right)_{q \times q}$.

Let $\Psi_{S_{i}}$ be the generalized Euler function ${ }^{[5]}$ on $S_{i}$. It can be defined the generalized Euler function on $S$ as

$\Psi_{S}\left(d_{j}\right)=d_{j}^{(1)} d_{j}^{(2)} \cdots d_{j}^{(n)}-\sum_{d_{i} p d_{j}, d_{i} \neq d_{j}} \Psi_{S}\left(d_{i}\right)$ where $d_{j}^{(1)} d_{j}^{(2)} \cdots d_{j}^{(n)}$ note the product of the component in $d_{j}$. Suppose

$\forall d_{i} \in S=\left\{d_{1}, d_{2}, \cdots, d_{q}\right\}, d_{i}=\left(d_{i}^{(1)}, d_{i}^{(2)}, \cdots, d_{i}^{(n)}\right)$.

We have gotten the GCD matrix on $S$ as follows : $\langle S\rangle_{q \times q}=\left(\operatorname{gcd}\left(d_{i}, d_{j}\right)\right)_{q \times q}$. 


\section{Main results}

$\forall 1 \leq i \leq n, T_{i}$ is the minimum GCDC ${ }^{[6]}$ set containing $S_{i}$. Let $T$ is MC set and $T=T_{1} \times T_{2} \times \cdots \times T_{n}=\left\{f_{1}, f_{2}, \cdots, f_{t}\right\}$, it is clearly to $T \supseteq S$. We have some conclusions as follows:

Theorem1 Let $S$ and $T$ be given above, then $\langle S\rangle=A A^{T}=E \Lambda E^{T}$,so $\langle S\rangle$ is the positive definite. Where $A=\left(a_{i j}\right), E=\left(e_{i j}\right)_{q x}$,

$\Lambda=\operatorname{diag}\left(\Psi_{T}\left(f_{1}\right), \Psi_{T}\left(f_{2}\right), \cdots, \Psi_{T}\left(f_{t}\right)\right) . \quad A^{T}$

and $E^{T}$ are respectively the transpose, $\Psi_{T}$ is the generalized Euler function on $T$. And $e_{i j}=\left\{\begin{array}{cc}1 & f_{i} p d_{i} \\ 0 & \text { athenwise }\end{array} a_{i j}=e_{i j}\left\{\Psi_{T}\left(f_{j}\right)\right\}^{1 / 2}\right.$

Theorem 2 Let $S, T$ and $E$ be given above, then $\operatorname{det}\langle S\rangle=$

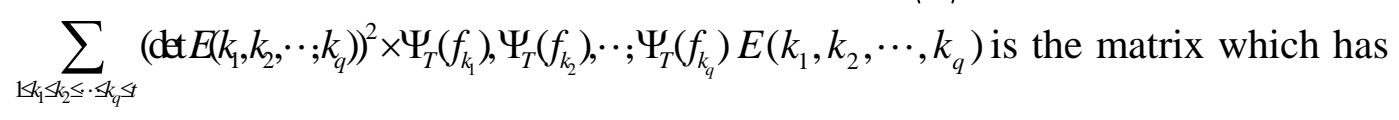
formed by $k_{1}$ th,$k_{2}$ th $, \cdots, k_{q}$ th columns taken out $E$ according to the original position.

We can discuss the upper or lower bound of the determinants for $\langle S\rangle$ in the following theorem:

Theorem 3 Let $S, T$ and $E$ be given above, then(1) $\operatorname{det}\langle S\rangle \geq \prod_{d \in S} \Psi_{T}(d)$, the equality holds if and only if $S$ is MC set and the element's divisor in $S$ has no existed in $T \backslash S$;

(2) $\operatorname{det}\langle S\rangle \leq \prod_{i=1}^{q}\left(d_{i}^{(1)} d_{i}^{(2)} \cdots d_{i}^{(n)}\right)$;

(3)When each $S_{i}$ is FC set, $\operatorname{det}\langle S\rangle=\prod_{\left(x_{1}, x_{2}, \cdots, x_{n}\right) \in S}\left[\phi\left(x_{1}\right) \phi\left(x_{2}\right) \cdots \phi\left(x_{n}\right)\right]$.

\section{Proofs of the main results}

In this section, we have mainly proved above theorems. If the positive integer set $S=\left\{x_{1}, x_{2}, \cdots, x_{n}\right\}$ is the GCDC set, then the generalized Euler function 
$\Psi_{S}(x) \geq \phi(x), \forall x \in S$. If $S$ is the FC set, then $\Psi_{S}(x)=\phi(x), \forall x \in S, S=T$, $V_{x_{j}}=\left\{z \in T: \min \{y \in S: z \mid y\}=x_{j}\right\}=\left\{x_{j}\right\}$ We have given the following lemma.

Lemma 1 Let $S$ and $T$ be given above, then $\Psi_{S}(x)=\prod_{i=1}^{n} \Psi_{S_{i}}\left(x_{i}\right)$.

Now, we have proved the main results as follows:

The proof of Theorem 1: Take

$\Lambda=\operatorname{diag}\left(\Psi_{T}\left(f_{1}\right), \Psi_{T}\left(f_{2}\right), \cdots, \Psi_{T}\left(f_{t}\right)\right)$, then $D=E \Lambda=\left(e_{i j} \Psi_{T}\left(f_{j}\right)\right)_{q \times t}$, So the

$(i, j)$-array in $E \Lambda E^{T}$ is

$\sum_{k} e_{i k} \Psi_{T}\left(f_{k}\right) e_{j k}=\sum_{k} e_{i k} e_{j k} \Psi_{T}\left(f_{k}\right)$

$=\sum_{f_{k} p d_{i}, f_{k} p d_{j}} \Psi_{T}\left(f_{k}\right)=\sum_{f_{k} p\left(d_{i}, d_{j}\right)_{p}} \Psi_{T}\left(f_{k}\right)$

$=\operatorname{gcd}\left(d_{i}, d_{j}\right)$.

So we have obtained $<S>=E \Lambda E^{T}$. By lemma 1, it is easy to $\Psi_{S}(x)=\prod_{i=1}^{n} \Psi_{S_{i}}\left(x_{i}\right)$.

From [8], $\forall 1 \leq i \leq n, T_{k}$ is GCDC set, but $\Psi_{T_{k}} \geq \varphi\left(y_{k}\right)>0$, therefore $\Psi_{T}(y)>0$.

Suppose $A=E \Lambda^{1 / 2}=$

$\operatorname{Ediag}\left(\left\{\Psi_{T}\left(f_{1}\right)\right\}^{1 / 2},\left\{\Psi_{T}\left(f_{2}\right)\right\}^{1 / 2}, \cdots,\left\{\Psi_{T}\left(f_{t}\right)\right\}^{1 / 2}\right)$, then $<S>=A A^{T}$, that is $<S>=A A^{T}=$

$E \Lambda E^{T}$. So is well to the theorem.

The proof of theorem 2: from the proof of Theorem 1 , it is easy to known that $<S>=E E^{T}$. Take $D=E \Lambda=\left(e_{i j} \Psi_{T}\left(f_{j}\right)\right)_{q \times t}$.

Use the Cauchy-Binet formula ${ }^{[9]}$ to $<S>=E \Lambda E^{T}$, the result is right.

The reason that the third equality has established is that each column has extracted the common divisor in the determinant. So the theorem 2 holds.

Corollary 1 If $S=S_{1} \times S_{2} \times \cdots \times S_{n}$ is MC set, then $\operatorname{det}[S]=\prod_{i=1}^{q} \Psi_{S}\left(d_{i}\right)$.

The proof of Theorem 3: By theorem 2, we have known $<S>=E \Lambda E^{T}$ and $\Lambda=\operatorname{diag}\left(\Psi_{T}\left(f_{1}\right), \Psi_{T}\left(f_{2}\right), \cdots, \Psi_{T}\left(f_{t}\right)\right)$.

Because $T_{k}$ is GCDC set, it is to know that $\Psi_{T_{k}} \geq \varphi\left(y_{k}\right)>0$, that is $\Psi_{T}(y)>0$ by 
proposition 1.Then $\langle S\rangle$ is positive definite. From theorem 2, $\operatorname{det}\langle S\rangle \geq$

$\prod_{d \in S} \Psi_{T}(d)=\prod_{i=1}^{q} \Psi_{T}\left(d_{i}\right) . \quad$ Suppose

$T=T_{1} \times T_{2} \times \cdots \times T_{n}=\left\{f_{1}, f_{2}, \cdots, f_{t}\right\}$, it is apparently to $t>q$.

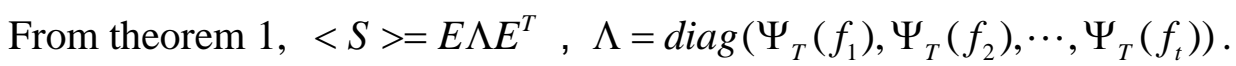

We have separate the matrix into the block $E=\left(E_{1}, E_{2}\right)$. Let

$\Lambda_{1}=\operatorname{diag}\left(\Psi_{T}\left(f_{1}\right), \Psi_{T}\left(f_{2}\right), \cdots, \Psi_{T}\left(f_{q}\right)\right)$

$\Lambda_{2}=\operatorname{diag}\left(\Psi_{T}\left(f_{q+1}\right), \Psi_{T}\left(f_{q+2}\right), \cdots, \Psi_{T}\left(f_{t}\right)\right)$. We have the equality $C=E_{1} \Lambda_{1} E_{1}^{T}$ and

$D=E_{2} \Lambda_{2} E_{2}^{T}$. So $<S>=E \Lambda E^{T}=C+D$

Because of $\Psi_{T}\left(d_{i}\right)>0, \forall d_{i} \in S$, we have known that $\Lambda_{1}$ is positive definite.

But det $C \neq 0$, it is positive definite to $C$ and positive semi-definite to $D$.

$\operatorname{det}\langle S\rangle=\operatorname{det}(C+D) \geq \operatorname{det} C+\operatorname{det} D \geq \operatorname{det} C$. That is $\operatorname{det}\langle S\rangle \geq \prod_{d \in S} \Psi_{T}(d)$, the equality has established if and only if $D=0$.

While $\Lambda_{2}$ is positive definite, there must be $E_{2}=0$, that is if and only if $S$ is MC set and the element's divisor in $S$ has no existed in $T \backslash S . T$ is the GCDC set, so is $S$. (1) is correct.

( 2 ) $\langle S\rangle$ is positive definite by theorem 2 , we have known that

$\operatorname{det}\langle S\rangle \leq \prod_{i=1}^{q} \operatorname{gcd}\left(d_{i}, d_{i}\right)=\prod_{i=1}^{q}\left(d_{i}^{(1)} d_{i}^{(2)} \cdots d_{i}^{(n)}\right) \quad(3)$ If $S$ is FC set, then $S=T$.We know $\Psi_{T_{k}}\left(y_{k}\right)=\phi\left(y_{k}\right)$, therefore

$\Psi_{T}(y)=\prod_{k=1}^{n} \Psi_{T_{k}}\left(y_{k}\right)=\prod_{k=1}^{n} \phi\left(y_{k}\right)$.So

$\operatorname{det}\langle S\rangle=\prod_{\left(x_{1}, x_{2}, \cdots, x_{n}\right) \in S}\left[\phi\left(x_{1}\right) \phi\left(x_{2}\right) \cdots \phi\left(x_{n}\right)\right]$, To sum up, (1) (2) (3) are all hold.

\section{The generalized GCD matrix}

Let $P_{1}, P_{2}, \cdots, P_{n}$ be the meet semi-lattice, $\forall 1 \leq i \leq n, p_{i}$ is the partially ordered relation of $P_{i}$. Let $P=P_{1} \times P_{2} \times \cdots \times P_{n}, \forall x, y \in P$ $(x, y)_{p}=\left(\left(x_{1}, y_{1}\right)_{p_{1}},\left(x_{2}, y_{2}\right)_{p_{2}}, \cdots,\left(x_{n}, y_{n}\right)_{p_{n}}\right)$.

Let $g_{i}: P_{i} \rightarrow R$ be the function who take the value in the commutative ring $R$. $\forall x=\left(x_{1}, x_{2}, \cdots ; x_{n}\right) \in P$ defined $g(x)=\prod_{i=1}^{n} g_{i}\left(x_{i}\right)$. 
Let $T_{i}$ be the smallest finite MC subset of $P_{i}$ include in $S_{i}$.

If $T$ is MC subset include in $S$ and

$\Psi_{S_{i}, g_{i}}>0$, then $\forall d=\left(d_{1}, d_{2}, \cdots, d_{n}\right) \in S$,

$\Psi_{S, g}\left(d_{j}\right)=\prod_{i=1}^{n} \Psi_{S_{i}, g_{i}}\left(d_{i}\right)$ and $\Psi_{S, g}>0$.

Theorem 4 Let $S$ and $T$ be given above, $T$ is a MC subset contained $S$, if $\Psi_{S, g}>0$, then (1) det $\langle S\rangle_{g} \geq \prod_{d \in S} \Psi_{T, g}(d)$; (2) det $\langle S\rangle_{g}<\prod_{d \in S} g(d)$.

The following theorem is not required $\Psi_{S, g}>0$.

Theorem 5 Let $S=S_{1} \times S_{2} \times \cdots \times S_{n}$, if $S$ is MC set, then we have the following conclusion: $\operatorname{det}\langle S\rangle_{g}=\prod_{d \in S} \Psi_{T, g}(d)$

As a result, Theorem 4 is similar to the theorem 3, and Theorem 5 is similar to the theorem 13 in [4], the proof omits.

We have gotten the certain relations between generalized GCD matrices and the generalized Euler function. It is also available to consider the invariant divisor and characteristic divisor or the eigenvalues of the greatest common divisor matrices for studying them, which is the next working target to us.

\section{References}

[1] S.Beslin and S.Ligh, Greatest common divisor matrices[J]. Linear Algebra Appl. 118 (1989):69-76.

[2] Z.Li, The determinants of GCD matrices[J]. Linear Algebra Appl. 134 (1990):137-143.

[3] K.Bourque and S.Ligh, Matrices associated with arithmetical functions[J]. Linear and Multilinear Algebra. 34 (1993):261-267.

[4] H.J.S.Smith, On the Value of a certain arithmetical determinant[J]. Proc. London Math.Soc. 7 (1875-1876):208-

212.

[5] S.Hong, Bounds for determinants of Matrices associated with classes of arithmetical functions[J]. Linear Algebra Appl. 281 (1998):311-322.

[6] Ismo.Korkee. Pentti Haukkanen, Bounds for determinants of meet matrices associated with incidence functions[J].Linear Algebra Appl. 329 (2001):77-88. 
[7] R.P Stanley, Enumerative combinatorics (Vol.I). Wadsworth and Brooks/cole.Monterey, California,1986

[8] N.Jacobson, Basic Algebra I [M].W.H.Freeman and company, San Francisco1974

[9] Fan Yun, Liu Hongwei, Group and Combination coding [M]. Wuhan University Press, Wuhan,China, 2002

Received: March 26, 2013 